\title{
Dose conversion from aminophylline to theophylline in preterm infants
}

\author{
J Reese, G Prentice, V Y H Yu
}

\begin{abstract}
Twenty two preterm infants were prospectively evaluated to assess the need for dose adjustment when converting enteral and parenteral routes of methylxanthine administration. Serum theophylline concentrations remained unchanged in 18 infants after conversion from intravenous aminophylline to theophylline by mouth without dose reduction, as is currently recommended. Intravenous aminophylline and theophylline by mouth may therefore be prescribed at equivalent doses, with a possible reduction in drug errors, and improved stability of serum concentrations.
\end{abstract}

(Arch Dis Child 1994; 71: F51-F52)

Theophylline has been used as a respiratory stimulant in infants since 1973. It is useful in weaning infants from mechanical ventilation, and is an effective drug in the treatment of bronchopulmonary dysplasia and apnoea of prematurity.

Serum concentrations must be monitored closely and dose alterations made with increasing postnatal age due to maturational changes in theophylline metabolism. ${ }^{1-4}$ Intravenous theophylline is administered as its ethylenediamine salt, aminophylline. The aminophylline dose used is usually 1.25 times the theophylline dose, as aminophylline is composed of approximately $80 \%$ theophylline. ${ }^{3}$ Therefore, when infants begin to tolerate enteral feeding and intravenous aminophylline is converted to theophylline by mouth, its dose is routinely reduced. Similarly, dose adjustment is required when enteral nutrition and theophylline by mouth are stopped for feed intolerance, and intravenous aminophylline is started.

A previous study in our nursery has shown that theophylline by mouth is $83 \%$ bioavailable. ${ }^{1}$ As a dose reduction of $20 \%$ would theoretically be required to change from intravenous aminophylline to theophylline by mouth, we postulated that oral bioavailability would reduce the amount of drug systemically available by approximately the same amount and no dose reduction would be necessary. Therefore, we prospectively evaluated whether serum theophylline concentrations were altered when infants were changed from intravenous aminophylline to theophylline by mouth without dose reduction, and vice versa.

\section{Patients and methods}

Serum theophylline concentrations were measured in infants in whom the same doses of theophylline by mouth and intravenous aminophylline were given. Those who received intravenous aminophylline for long enough to achieve a steady state concentration (for this study, considered to be four days ${ }^{24}$ ) and who were subsequently changed to theophylline by mouth for a similar period were evaluated. Patients were excluded if they concurrently received erythromycin, phenobarbitone, phenytoin, cimetidine, or other drugs suspected of altering theophylline metabolism. ${ }^{5}$ Similarly, infants with asphyxia were excluded due to suspicion of altered theophylline clearance rates. ${ }^{2}$

Intravenous aminophylline (David Bull Laboratories) was given as a $10 \mathrm{mg} / \mathrm{kg}$ loading dose over 15 minutes, and then a maintenance treatment of $2.5 \mathrm{mg} / \mathrm{kg}$ every 12 hours, begun 12 hours after the loading dose. When the infant tolerated enteral feeding, theophylline by mouth (Elixophylline, Schering) was ordered at the same dose and frequency as the most recent aminophylline prescription. Doses by mouth were given at the same time as milk feeds.

Serum theophylline concentrations were determined 30 minutes before a dose by dry chemical assay (Ektachem 700, Eastman Kodak Company, Rochester, NY). An acceptable therapeutic range for our assay was 45-90 $\mu \mathrm{mol} / 1(8-16 \mu \mathrm{g} / \mathrm{ml})$, with a desired concentration of $70 \mu \mathrm{mol} / \mathrm{l}(12.5 \mu \mathrm{g} / \mathrm{ml})$. Data were analysed using Student's $t$ test (paired, two tailed).

\section{Results}

Eighteen infants were changed from intravenous aminophylline to theophylline by mouth at equivalent doses and met the steady state criteria for both routes. A second set of measurements were included in one infant who was changed from the intravenous to oral route on a second occasion. Five additional infants who met the steady state criteria when the drugs were changed from the oral to intra-
Mean (SD)

$1216(682-1889)$

$28 \cdot 5(1 \cdot 9)$

$21(16)$

$15(3-54)$

$31 \cdot 1(27 \cdot 1-37 \cdot 6)$

$846(190)$

$26 \cdot 8(0 \cdot 8)$

$7(26-28)$

$29(20-177)$

$31(28 \cdot 9-52 \cdot 3)$ 


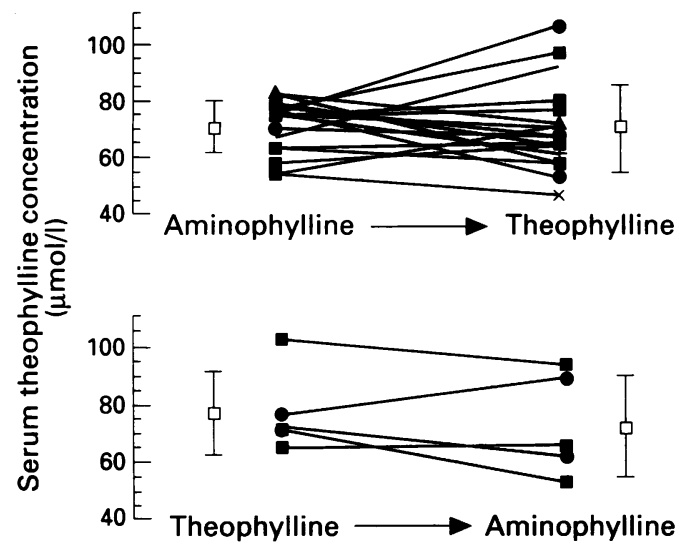

Theophylline serum concentrations during intravenous administration and administration by mouth. Upper panel: conversion from intravenous aminophylline to theophylline by mouth (open boxes represent means, with SD bars). Lower panel: conversion from by mouth to intravenous route of administration.

venous route were also studied. The table gives the patient characteristics.

There was no significant difference between mean serum theophylline concentrations while receiving intravenous aminophylline and after conversion to theophylline by mouth (70.9 (9.0) $v 70.5(15 \cdot 2) \mu \mathrm{mol} / \mathrm{l} ; \mathrm{p}=0.92)$ (fig). No infant showed clinical signs of toxicity while receiving the drug intravenously or by mouth. When controlled for postnatal age, five infants who had measurements taken while receiving the drug intravenously or by mouth before 3 weeks of age showed no difference between mean serum values $(p=0.94)$, nor did eight remaining infants with both measurements taken after three weeks $(p=0 \cdot 60)$. Eight infants showed an increase and 10 infants showed a decrease in serum concentrations after changing to receiving the drug by mouth. There was also no significant difference between mean serum theophylline concentrations while receiving theophylline by mouth and after conversion to intravenous aminophylline $(77 \cdot 2$ $(14.4) v 72.6(17.5) \mu \mathrm{mol} / \mathrm{l} ; \mathrm{p}=0.44)$ (fig).

\section{Discussion}

Theophylline has been reported to be one of the paediatric drugs most frequently associated with prescription errors. ${ }^{6}$ Owing to alterations in the tolerance of enteral feeding in the neonatal period, the oral and intravenous routes of administration are frequently interchanged. As theophylline has potentially serious side effects and a narrow therapeutic window, ${ }^{35}$ our earlier practice has been to reduce the theophylline dose by mouth to $80 \%$ of the aminophylline dose, based on the chemical composition of the drug. The bioavailability of drugs given by mouth, however, depends on factors such as $\mathrm{pH}$, microbial flora, and gastric motility. ${ }^{7}$ Older infants and children are assumed to have $100 \%$ absorption of theophylline given by mouth. It was suggested that $100 \%$ bioavailability was possible for theophylline by mouth in preterm infants, ${ }^{4}$ but other studies have found it to be approximately $80 \%$ bioavailable. ${ }^{13}$ The bioavailability of aminophylline from rectal suppositories is similarly $70-80 \%{ }^{8}$ The present study showed that serum theophylline concentrations remained constant after changing from the intravenous to the oral route without dose adjustment. No toxicity was noted, as might be expected if theophylline by mouth was $100 \%$ bioavailable. In addition, data from a small number of patients showed that conversion from oral to intravenous administration may also be carried out without altering the drug dose.

Whether enteral feeding alters the bioavailability of theophylline given by mouth is unclear. A delayed absorption of theophylline was noted when given with enteral feeds, but only the rate and not the total amount was affected. ${ }^{7}$

Although current recommendations are an alteration in dose when converting between intravenous and oral administration, our data support the hypothesis that intravenous aminophylline may be converted to oral theophylline without a reduction in dose. In preterm infants, whose clinical status is often labile, this policy should help reduce prescription errors and ensure more predictable serum theophylline concentrations despite frequent changes between enteral and parenteral drugs.

$1 \mathrm{Yu}$ VYH. The year in neonatology. Med $\mathcal{f}$ Aust 1980; i: 198-203.

2 Gillman JT, Gal P, Levine RS, Hersh CB, Erkan NV. Factors influencing theophylline disposition in 179 newborns. Ther Drug Monit 1986; 8: 4-10.

3 Aranda IV, Turmen T. Methylxanthines in apnea of prematurity. Clin Perinatol 1979; 6: 87-108.

4 Moore ES, Faix RG, Banagle RC, Grasela TH. The population pharmacokinetics of theophylline in neonates and young infants. F Pharm Biopharm 1989; 17: 47.

5 Kauffman RE, Banner W, Berlin C, et al. American Academy of Pediatrics, Committee on Drugs. Precautions concerning the use of theophylline. Pediatrics 1992; 89: 781-3.

6 Folli HL, Poole RL, Benitz WE, Russo JC. Medication error prevention by clinical pharmacists

7 Heimann G, Murgescu J, Bergt U. Influence of food intake on bioavailability of theophylline in premature infants. Eur on bioavailability of theophylline in

8 Karlson MO, Thomson AH, McGovern EM, Chow P, Evans TJ, Kelman AW. Population pharmacokinetics of rectal theophylline in neonates. Ther Drug Monit 1991; 13: 195-200. 


\section{Correction: Dose conversion from aminophylline to theophylline in preterm infants}

Reese J, Prentice G, Yu VJ. Dose conversion from aminophylline to theophylline in preterm infants (Arch Dis Child Fetal Neonatal Ed 1994;71:F51-52.)

The original online version of this article included the following incorrect sentence in its abstract: 'Serum theophylline concentrations remained unchanged in 18 infants after conversion from intravenous aminophylline to theophylline by mouth with dose reduction, as is currently recommended'.
This is incorrect and should have read 'Serum theophylline concentrations remained unchanged in 18 infants after conversion from intravenous aminophylline to theophylline by mouth without dose reduction, as is currently recommended', as it does in the abstract of the pdf version. This has now been corrected.

(C) Article author(s) (or their employer(s) unless otherwise stated in the text of the article) 2019. All rights reserved. No commercial use is permitted unless otherwise expressly granted.

Arch Dis Child Fetal Neonatal Ed 2019;104:F570. doi:10.1136/fn.71.1.f51 corr1

Deck for updates 